Pacific Journal of Mathematics

BEANO DERIVATIVES AND GENERAL INTEGRALS

Routhcott Bulled and S. N. MukHopadhyay 


\section{PEANO DERIVATIVES AND GENERAL INTEGRALS}

\section{P. S. Bullen and S. N. Mukhopadhyay}

It is known that if a function $f$ has a finite derivative (or approximate derivative) on a set $E$ on which $f$ is continuous then $f$ is $A C G_{*}$ (or $A C G$ ) on $E$ and that if $f$ is $A C G_{*}$ (or $\left.A C G\right)$ on a set $E$ then a finite derivative (or approximate derivative) of $f$ exists almost everywhere in $E$. These results are extended by Sargent in the case of generalized derivatives of higher order. She has proved that if $f_{n+1}$, the generalized derivative of $f$ of order $n+1$, exists in an interval $[a, b]$ then the derivative $f_{n}$ is $V_{n}-A C G^{*}$ on $[a, b]$ and that if $f_{n}$ is $V_{n}-A C G^{*}$ on $[a, b]$ then $f_{n+1}$ exists and is equal to the approximate derivative of $f_{n}$ almost everywhere in $[a, b]$.

The present work is concerned with extending still further these results of Sargent by introducing a more general definitions of absolute continuity for the $n$th derivatives. It also introduces an approximate $P^{n}$-integral which generalizes the $P^{n}$-integral of James and Bullen.

1. Definitions and notations. Let $f$ be defined and finite in some neighborhood $N$ of a point $x_{0}$. If

$$
f(x)=\sum_{r=0}^{n} \frac{\left(x-x_{0}\right)^{r}}{r !} \alpha_{r}+o\left(\left(x-x_{0}\right)^{n}\right)
$$

as $x \rightarrow x_{0}$, where $\alpha_{0}=f\left(x_{0}\right)$ and $\alpha_{1}, \alpha_{2}, \cdots \alpha_{n}$ are finite and independent of $x$ but depends on $x_{0}$ only, then $\alpha_{r}$ is called the $r$ th Peano derivative (also called $r$ th de la Vallee Poussin derivative [4]) of $f$ at $x_{0}$ and is denoted by $f_{r}\left(x_{0}\right)$. From the definition it follows that if $f_{n}\left(x_{0}\right)$ exists, then $f_{r}\left(x_{0}\right)$ exists for $0 \leqq r \leqq n$. Also if the ordinary $n$th derivative $f^{(n)}\left(x_{0}\right)$ exists then $f_{n}\left(x_{0}\right)$ exists and equals $f^{(n)}\left(x_{0}\right)$ and so $f_{r}\left(x_{0}\right)$ also exists and equals $f^{(r)}\left(x_{0}\right)$ for $0 \leqq r \leqq n$. The converse is true only for $n=1$.

Let us suppose that $f_{n}\left(x_{0}\right)$ exists. Then the upper and the lower $(n+1)$ th Peano derivative of $f$ at $x_{0}$ are defined to be the upper and the lower limits of

$$
\frac{(n+1) !}{h^{n+1}}\left\{f(x)-\sum_{r=0}^{n} \frac{\left(x-x_{0}\right)^{r}}{r !} \alpha_{r}\right\}
$$

as $x \rightarrow x_{0}$ and will be denoted by $\bar{f}_{n+1}\left(x_{0}\right)$ and $\underline{f}_{n+1}\left(x_{0}\right)$ respectively. Further, by restricting $x$ suitably one can define one sided Peano derivatives of $f$ at $x_{0}$ and denoted by $\bar{f}_{n+1}^{+}\left(x_{0}\right), \underline{f}_{n+1}^{-}\left(x_{0}\right)$, etc.

The approximate upper and approximate lower $(n+1)$ th Peano 
derivatives of $f$ at $x_{0}$ are defined to be the approximate upper and the approximate lower limits of the expression (1.2) as $x \rightarrow x_{0}$ and are denoted by $\bar{f}_{n+1, a p}\left(x_{0}\right), f_{n+1, a p}\left(x_{0}\right)$ respectively.

If $f_{n}\left(a_{0}\right)$ exists we can write from (1.1)

$$
f(x)=\sum_{r=0}^{n} \frac{\left(x-x_{0}\right)^{r}}{r !} f_{r}(x)+\frac{\left(x-x_{0}\right)^{n}}{n !} \varepsilon_{n}\left(x_{0}, x\right)
$$

which gives

$$
\varepsilon_{n}\left(x_{0}, x\right)=\frac{n !}{\left(x-x_{0}\right)^{n}}\left\{f(x)-\sum_{r=0}^{n} \frac{\left(x-x_{0}\right)^{r}}{r !} f_{r}\left(x_{0}\right)\right\} .
$$

Then $\varepsilon_{n}\left(x_{0}, x\right)$ is defined for all $x \in N$ except for $x=x_{0}$. We complete the definition by setting $\varepsilon_{n}\left(x_{0}, x_{0}\right)=0$. Then $\varepsilon_{n}\left(x_{0}, x\right)$ is defined throughout $N$ and is a continuous function of $x$ when $f$ is continuous.

Let $f$ be defined in the interval $[a, b]$ and let $a \leqq c<d \leqq b$. If $f_{n}(c)$ and $f_{n}(d)$ exist we define

$$
\begin{aligned}
& \bar{\omega}_{n}(f ; c, d)=\max \left[\sup _{c \leqq x \leqq d} \varepsilon_{n}(c, x), \sup _{c \leqq x \leqq d}\left\{-\varepsilon_{n}(d, x)\right\}\right] \\
& \underline{\omega}_{n}(f ; c, d)=\min \left[\inf _{c \leqq x \leqq d} \varepsilon_{n}(c, x), \inf _{c \leqq x \leqq d}\left\{-\varepsilon_{n}(d, x)\right\}\right] \\
& \omega_{n}(f ; c, d)=\bar{\omega}_{n}(f ; c, d)-\underline{\omega}_{n}(f ; c, d) .
\end{aligned}
$$

Since $\underline{\omega}_{n}(f ; c, d) \leqq 0 \leqq \bar{\omega}_{n}(f ; c, d)$, we have $\omega_{n}(f ; c, d) \geqq 0$. If there is no confusion we shall simply write $\bar{\omega}_{n}(c, d)$ etc. to denote $\bar{\omega}_{n}(f ; c, d)$ etc.

Let us suppose that $f_{n}$ exists on a set $E \subset[a, b]$. Then $f$ is said to be $A C_{n}^{*}$ below (resp. above), written $A C_{n}^{*}$ (resp. $\overline{A C}_{n}^{*}$ ) on $E$ if for every $\varepsilon>0$ there is a $\delta>0$ such that

$$
\sum_{i} \underline{\omega}_{n}\left(a_{i}, b_{i}\right)>-\varepsilon\left(\operatorname{resp} . \sum_{i} \bar{\omega}_{n}\left(a_{i} b_{i}\right)<\varepsilon\right)
$$

for every sequence of non overlapping intervals $\left\{\left(a_{i}, b_{i}\right)\right\}$ whose end points are in $E$ and

$$
\sum_{i}\left(b_{i}-a_{i}\right)<\delta
$$

If $f$ is both $\overline{A C}_{n}^{*}$ and $\underline{A C}_{n}^{*}$ on $E$ then $f$ is said to be $A C_{n}^{*}$ on $E$. It is clear from the definition that $f$ is $A C_{n}^{*}$ on $E$ iff for every $\varepsilon>0$ there is $\delta>0$ such that

$$
\sum_{i} \omega_{n}\left(a_{i}, b_{i}\right)<\varepsilon
$$

for every sequence of non overlapping intervals $\left\{\left(a_{i}, b_{i}\right)\right\}$ with end points in $E$ and

$$
\sum\left(b_{i}-a_{i}\right)<\delta
$$


The function $f$ is said to be $A C_{n} G^{*}$ below (resp. above) written $\underline{A C_{n} G^{*}}$ (resp. $\overline{A C_{n} G^{*}}$ ) on $E$ if $E$ can be expressed as a countable union of sets on each of which $f$ is $A C_{n}^{*}$ (resp. $\overline{A C_{n}^{*}}$ ). The function $f$ is $A C_{n} G^{*}$ on $E$ if $E$ is a countable union of sets on each of which $f$ is $A C_{n}^{*}$. Clearly $f$ is $A C_{n} G^{*}$ on $E$ iff $f$ is both ${\overline{A C_{n}}}^{*}$ and $A C_{n} G^{*}$ on $E$. We shall use the notation $\left[A C_{n} G^{*}\right]$ etc. instead of $A C_{n} \overline{G^{*} \text { etc. }}$ to mean that the set $E$ can be expressed as a countable union of closed sets on each of which $f$ is $A C_{n}^{*}$. For a function $f$ to be $A C, A C_{*}$ etc. on a set $E$ we refer to the definition given in [7]. Since $f_{n}$ is the function $f$ when $n=0, A C_{0}^{*}$ is the same as $A C_{*}$ and $A C_{0} G^{*}$ is the same as $A C G_{*}$ in [7].

The function $f$ is $A C$ below (resp. above) written $\underline{A C}$ (resp. $\overline{A C}$ ) on $E$ if for every $\varepsilon>0$ there is $\delta>0$ such that

$$
\sum_{i}\left\{f\left(b_{i}\right)-f\left(a_{i}\right)\right\}>-\varepsilon\left(\operatorname{resp} . \sum_{i}\left\{f\left(b_{i}\right)-f\left(a_{i}\right)\right\}<\varepsilon\right)
$$

for every sequence of non overlapping intervals $\left\{\left(a_{i} b_{i}\right)\right\}$ whose end points are in $E$ and

$$
\sum_{i}\left(b_{i}-a_{i}\right)<\delta .
$$

The function $f$ is $[\overline{A C G}]$ or $[A C G]$ on $E$ according as $E$ can be expressed as a countable union of closed sets on each of which $f$ is $\overline{A C}$ or $\underline{A C}$ and $f$ is $[A C G]$ if and only if $f$ is both $[\overline{A C G}]$ and [ACG].

Throughout, $\mu(A)$ will denote the Lebesgue measure of the measurable set $A$ and the approximate derivative and the Dini derivatives of $f$ are denoted by $f_{a p}^{\prime}$ and $D^{+} f$, etc.

\section{Preliminary lemmas.}

Lemma 1. Let $f_{n}$ exist and be bounded above on $[a, b]$. If $f$ is $\underline{A C_{n}^{*}}$ on $E \subset[a, b]$ then $f_{n}$ is $\underline{A C}$ on $E$.

Proof. Let $\sup f_{n}=M$. We suppose $M=0$. Then since $f$ is $\underline{A C}_{n}^{*}$ on $E$, given $\varepsilon>0$ there is $\delta>0$ such that

$$
\sum_{i} \underline{\omega}_{n}\left(a_{i}, b_{i}\right)>-\frac{\varepsilon}{2}
$$

for every sequence of non overlapping intervals $\left\{\left(a_{i}, b_{i}\right)\right\}$ with end points in $E$ satisfying

$$
\sum_{i}\left(b_{i}-a_{i}\right)<\delta
$$

Now 


$$
\frac{\left(b_{i}-a_{i}\right)^{n-1}}{(n-1) !} \varepsilon_{n-1}\left(f ; a_{i} b_{i}\right)=\frac{\left(b_{i}-a_{i}\right)^{n}}{n !} f_{n}\left(a_{i}\right)+\frac{\left(b_{i}-a_{i}\right)^{n}}{n !} \varepsilon_{n}\left(f ; a_{i}, b_{i}\right)
$$

i.e.,

$$
\frac{n}{b_{i}-a_{i}} \varepsilon_{n-1}\left(f ; a_{i}, b_{i}\right)-f_{n}\left(a_{i}\right)=\varepsilon_{n}\left(f ; a_{i}, b_{i}\right) \text {. }
$$

Similarly

$$
\frac{n}{b_{i}-a_{i}} \varepsilon_{n-1}\left(f ; b_{i}, a_{i}\right)+f_{n}\left(b_{i}\right)=-\varepsilon_{n}\left(f ; b_{i}, a_{i}\right) \text {. }
$$

Hence

$$
\begin{aligned}
f_{n}\left(b_{i}\right) & -f_{n}\left(a_{i}\right)=\varepsilon_{n}\left(f ; a_{i}, b_{i}\right)-\varepsilon_{n}\left(f ; b_{i}, a_{i}\right) \\
& -\left[\frac{n}{b_{i}-a_{i}} \varepsilon_{n-1}\left(f ; a_{i}, b_{i}\right)+\frac{n}{b_{i}-a_{i}} \varepsilon_{n-1}\left(f ; b_{i}, a_{i}\right)\right] .
\end{aligned}
$$

Since $f_{n}$ satisfies the mean value property [5] there are points $\xi_{i}$ and $\xi_{i}^{\prime}$ in $\left(a_{i}, b_{i}\right)$ such that

$$
\begin{aligned}
& \frac{n}{b_{i}-a_{i}} \varepsilon_{n-1}\left(f ; a_{i}, b_{i}\right)=f_{n}\left(\xi_{i}\right), \\
& \frac{n}{b_{i}-a_{i}} \varepsilon_{n-1}\left(f ; b_{i}, a_{i}\right)=f_{n}\left(\xi_{i}^{\prime}\right) .
\end{aligned}
$$

Since $f_{n}(x) \leqq 0$ for all $x$ in $[a, b]$, from (2.3) and (1.5)

$$
\begin{aligned}
f_{n}\left(b_{i}\right)-f_{n}\left(a_{i}\right) & \geqq \varepsilon_{n}\left(f ; a_{i}, b_{i}\right)-\varepsilon_{n}\left(f ; b_{i}, a_{i}\right) \\
& \geqq 2 \underline{\omega}_{n}\left(a_{i}, b_{i}\right) .
\end{aligned}
$$

Hence from (2.1)

$$
\sum_{i}\left\{f_{n}\left(b_{i}\right)-f_{n}\left(a_{i}\right)\right\} \geqq 2 \sum_{i} \underline{\omega}_{n}\left(a_{i} b_{i}\right)>-\varepsilon
$$

whenever the intervals $\left\{\left(a_{i}, b_{i}\right)\right\}$ satisfy (2.2). This shows that $f_{n}$ is $\underline{A C}$ on $E$.

If $M \neq 0$, consider $g(x)=f(x)-M\left(x^{n} / n !\right)$.

Then $g_{r}(x)=f_{r}(x)-M\left(x^{n-r} /(n-r) !\right), r=0,1, \cdots n$. So, $\sup g_{n}=\sup f_{n}-M=0$. Also $g$ is $\underline{A C}_{n}^{*}$ on $E$ and hence by the above argument $g_{n}$ is $\underline{A C}$ on $E$. Since $g_{n}=f_{n}-M, f_{n}$ is $\underline{A C}$ on $E$.

LEMma 2. Let $f$ be defined in $[a, b]$ and let $f_{n}$ exist on the closure $\bar{E}$ of $a$ set $E \subset[a, b]$. If $f$ is $A C_{n}^{*}$ on $E$ then $f$ is $A C_{n}^{*}$ on $\bar{E}$.

For proof we refer to an analogous Lemma of Sargent [8]. 
LEMMA 3. Let $f$ be defined in $[a, b]$ and let $f_{n}(c)$ and $f_{n}(d)$ exist where $a \leqq c<d \leqq b$. Then

$$
\left|f_{n}(d)-f_{n}(c)\right| \leqq K \omega_{n}(c, d)
$$

where $K$ is a constant depending on $n$ only.

Proof. As in [8] putting $h=(d-c) / n$ we have

$$
f_{n}(d)-f_{n}(c)=\sum_{r=0}^{n}(-1)^{n-r}\left(\begin{array}{l}
n \\
r
\end{array}\right) \frac{r^{n}}{n !}\left\{\varepsilon_{n}(c, c+r h)-\varepsilon_{n}(d, d-r h)\right\} .
$$

Denoting by $\Sigma^{(+)}$the summation over the terms in the right hand side of (2.5) for which $\varepsilon_{n}(c, c+r h)-\varepsilon_{n}(d, d-r h)$ is positive and by $\Sigma^{(-)}$for which it is negative and noticing that

$$
2 \underline{\omega}_{n}(c, d) \leqq \varepsilon_{n}(c, c+r h)-\varepsilon_{n}(d, d-r h) \leqq 2 \bar{\omega}_{n}(c, d), r=0,1, \cdots n
$$

we get from (2.5)

$$
\begin{aligned}
f_{n}(d)-f_{n}(c)= & \sum^{(+)}(-1)^{n-r}\left(\begin{array}{l}
n \\
r
\end{array}\right) \frac{r^{n}}{n !}\left\{\varepsilon_{n}(c, c+r h)-\varepsilon_{n}(d, d-r h)\right\} \\
& +\Sigma^{(-)}(-1)^{n-r}\left(\begin{array}{l}
n \\
r
\end{array}\right) \frac{r^{n}}{n !}\left\{\varepsilon_{n}(c, c+r h)-\varepsilon_{n}(d, d-r h)\right\} \\
& \leqq \Sigma^{(+)}\left(\begin{array}{l}
n \\
r
\end{array}\right) \frac{r^{n}}{n !}\left\{2 \bar{\omega}_{r}(c, d)\right\}+\Sigma^{(-)}\left(\begin{array}{l}
n \\
r
\end{array}\right) \frac{r^{n}}{n !}\left\{-2 \underline{\omega}_{n}(c, d)\right\} \\
& \leqq 2 \Sigma^{(+)}\left(\begin{array}{l}
n \\
r
\end{array}\right) \frac{r^{n}}{n !} \omega_{n}(c, d)+2 \Sigma^{(-)}\left(\begin{array}{l}
n \\
r
\end{array}\right) \frac{r^{n}}{n !} \omega_{n}(c, d) \\
& \leqq 4 \omega_{n}(c, d) \sum_{r=0}^{n}\left(\begin{array}{l}
n \\
r
\end{array}\right) \frac{r^{n}}{n !}=K \omega_{n}(c, d)
\end{aligned}
$$

where

$$
K=4 \sum_{r=0}^{n}\left(\begin{array}{l}
n \\
r
\end{array}\right) \frac{r^{n}}{n !}
$$

The other inequality can be similarly proved.

CoRollary 4. Let $f$ be defined in $[a, b]$ and let $f_{n}$ exist in $E \subset$ $[a, b]$. If $f$ is $A C_{n}^{*}$ on $E$ then $f$ is absolutely continuous on $E$.

LemMa 5. Let $f$ be defined in $[a, b]$ and let $f_{n}$ exist on a closed set $E \subset[a, b]$. If $f$ is $A C_{n}^{*}$ on $E$ then there exists a function $\lambda$ in $[a, b]$ with the following property:

(i) $\lambda^{(n)}$ exists and is continuous in $[a, b]$

(ii) $\lambda^{(n)}$ is $A C^{*}$ on $E$, and 
(iii)

$$
\lambda^{(r)}(x)=f_{r}(x) \text { for } x \in E ; r=0,1, \cdots n
$$

Proof. We define a function $g$ in $[a, b]$ such that $g=f_{n}$ on $E$ and $g$ is linear and continuous in the closure of each contiguous intervals of $E$. Then since $f$ is $A C_{n}^{*}$ on $E, f_{n}$ is absolutely continuous on $E$ by Corollary 4 , and hence $g$ is continuous in $[a, b]$ and is $A C^{*}$ on $E$. Let $\lambda$ be the function obtained by taking the indefinite integral of $g$ of order $n$ in $[a, b]$. Then $\lambda$ satisfies the requirements (i), (ii) and $\lambda^{(n)}(x)=f_{n}(x)$ for $x \in E$; the rest follows by an induction method due to Marcinkiewicz (Fund. Math. 27 (1937), p. 48-50; see also [8]).

Lemma 6. The function $\lambda$ defined in Lemma 5 is $A C_{n}^{*}$ on $E$.

Proof. Let $c$ and $d, c<d$, be any two points in $E$. Then for $c<x \leqq d$ we have

$$
\begin{aligned}
\varepsilon_{n}(\lambda ; c, x) & =\frac{n !}{(x-c)^{n}}\left\{\lambda(x)-\lambda(c)-\cdots-\frac{(x-c)^{n}}{n !} \lambda^{(n)}(c)\right\} \\
& =\lambda^{(n)}(\xi)-\lambda^{(n)}(c), c<\xi<x .
\end{aligned}
$$

Hence

$$
\begin{aligned}
\left|\varepsilon_{n}(\lambda ; c, x)\right| & =\left|\lambda^{(n)}(\xi)-\lambda^{(n)}(c)\right| \\
& \leqq 0\left(\lambda^{(n)} ; c, d\right)
\end{aligned}
$$

where $0\left(\lambda^{(n)} ; c, d\right)$ denotes the oscillation of $\lambda^{(n)}$ in $[c, d]$. Similarly for $c \leqq x<d$

$$
\left|\varepsilon_{n}(\lambda ; d, x)\right| \leqq 0\left(\lambda^{(n)} ; c, d\right)
$$

Thus

$$
\omega_{n}(\lambda ; c, d) \leqq 20\left(\lambda^{(n)} ; c, d\right) .
$$

Since $\lambda^{(n)}$ is $A C^{*}$ on $E$ by Lemma 5, it follows from (2.6) that $\lambda$ is $A C_{n}^{*}$ on $E$.

Lemma 7. If $f$ is a function of Baire class 1 in $[a, b]$ then for any perfect set $P \subset[a, b]$ there is a portion of $P$ on which $f$ is bounded.

This follows from the fact that $P$ contains a point of continuity of $f$ relative to $P$.

Lemia 8. If $f$ is $\underline{A C}$ on $[a, b]$ and $D^{+} f \geqq 0$ almost everywhere in $[a, b]$ then $f$ is nondecreasing in $[a, b]$.

Proof. Since $f$ is $\underline{A C}$ on $[a, b]$, for any $\varepsilon>0$ there is $\delta>0$ such 
that

$$
\sum_{i}\left\{f\left(b_{i}\right)-f\left(a_{i}\right)\right\}>-\varepsilon
$$

for every sequence of non overlapping intervals $\left\{\left(a_{i}, b_{i}\right)\right\}$ with the property

$$
\sum_{i}\left(b_{i}-a_{i}\right)<\delta .
$$

Let $E=\left\{x: x \in[a, b] ; D^{+} f(x) \geqq 0\right\}$. Then $\mu(E)=b-a$ and for $x \in E$ there is a sequence $\left\{h_{k}\right\}$ such that $h_{k} \rightarrow 0+$ and

$$
\frac{f\left(x+h_{k}\right)-f(x)}{h_{k}}>-\varepsilon, \quad k=1,2 \cdots
$$

Let $\mathscr{M}$ be the family of closed intervals $\left\{\left[x, x+h_{k}\right]: x \in E ; k=1,2\right.$, $\cdots\}$. Then $E$ is covered by $\mathscr{C}$ in the sense of Vitali. So, there is a finite number of non overlapping intervals in $\mathscr{M}$,

$$
\left[x_{1}, x_{1}^{\prime}\right],\left[x_{2}, x_{2}^{\prime}\right], \cdots,\left[x_{m}, x_{m}^{\prime}\right]
$$

say, such that

$$
\mu\left(E-\bigcup_{i=1}^{m}\left[x_{i}, x_{i}^{\prime}\right]\right)<\delta
$$

i.e. ,

$$
\mu\left([a, b]-\bigcup_{i=1}^{m}\left[x_{i}, x_{i}^{\prime}\right]\right)<\delta .
$$

From (2.8) and (2.7) we deduce

$$
\sum_{i=1}^{m+1}\left\{f\left(x_{i}\right)-f\left(x_{i-1}^{\prime}\right)\right\}>-\varepsilon
$$

where $a=x_{0}^{\prime}$ and $b=x_{m+1}$. Also from $\left(2.7^{\prime}\right)$

$$
f\left(x_{i}^{\prime}\right)-f\left(x_{i}\right)>-\varepsilon\left(x_{i}^{\prime}-x_{i}\right), \quad i=1,2, \cdots .
$$

Hence from (2.9) and (2.10)

$$
f(b)-f(a)>-\varepsilon-\varepsilon(b-a) .
$$

Since $\varepsilon>0$ is arbitrary, $f(a) \leqq f(b)$. Applying this argument to any subinterval, the proof is complete.

LEMMA 9. If $f_{n}$ is $\underline{A C}$ in $[a, b]$ and $\bar{f}_{n+1}^{+} \geqq 0$ almost everywhere in $[a, b]$ then $f_{n}$ is nondecreasing in $[a, b]$.

Proof. Let $Q$ be the set of all points $x$ in $[a, b]$ such that there 
is a neighborhood of $x$ in which $f_{n}$ is bounded. Then $Q$ is an open set. Let $(c, d)$ be any component interval of $Q$ and let $c<\alpha<\beta<d$. Then $f_{n}$ is bounded in $[\alpha, \beta]$ and hence it is the ordinary $n$th derivative $f^{(n)}$ in $[\alpha, \beta][5]$ and so all the previous derivatives $f^{(1)}, f^{(2)}, \cdots$, $f^{(n-1)}$ exist and are continuous in $[\alpha, \beta]$. Also for any $x \in[\alpha, \beta]$, if $x+h \in[\alpha, \beta]$, we have

$$
\frac{(n+1) !}{h^{n+1}}\left\{f(x+h)-\sum_{k=0}^{n} \frac{h^{k}}{k !} f_{k}(x)\right\}=\frac{1}{\theta h}\left\{f_{n}(x+\theta h)-f_{n}(x)\right\}
$$

for some $\theta, 0<\theta<1$. Hence it follows that

$$
D^{+} f_{n}(x) \geqq \bar{f}_{n+1}^{+}(x) \text {. }
$$

Thus the relation (2.11) holds for all $x$ in $[\alpha, \beta]$. Since $f_{n}$ is $\underline{A C}$ in $[\alpha, \beta]$, by Lemma $8, f_{n}$ is nondecreasing $[\alpha, \beta]$. Since $[\alpha, \beta]$ is arbitrary, $f_{n}$ is nondecreasing in $(c, d)$. By the Darboux property of $f_{n}$ [5] we conclude that $f_{n}$ is continuous and nondecreasing in the closed interval $[c, d]$.

Let $H=[a, b]-Q$. Then $H$ is closed. Also $H$ has no isolated point. For, if $x_{0}$ is an isolated point of $H$ then there are points $p$ and $q$ such that $p<x_{0}<q$ and $f_{n}$ is nondecreasing and continous in the intervals $\left[p, x_{0}\right]$ and $\left[x_{0}, q\right]$ and so $f_{n}$ is nondecreasing and continuous in the interval $[p, q]$ contradicting the fact that $x_{0} \in H$. Thus $H$ is perfect. We shall show that $H$ is empty.

If possible suppose that $H$ is nonempty. Since $f_{n}$ is a function of Baire class 1 [5] by Lemma 7 there is a portion of $H$, say $[\alpha, \beta] \cap$ $H$ such that $f_{n}$ is bounded on $[\alpha, \beta] \cap H$. Since $f_{n}$ is monotone and continuous in the closure of each complementary intervals of $H$ in $[\alpha, \beta], f_{n}$ is bounded in $[\alpha, \beta]$. But this is a contradiction since $[\alpha, \beta]$ contains points of $H$. This completes the proof.

LEMmA 10. Let $f_{n}$ be $\left[\underline{A C G]}\right.$ on $[a, b]$ and let $\bar{f}_{n+1}^{+} \geqq 0$ almost everywhere in $[a, b]$. If $P$ is any perfect set on $[a, b]$ such that $f_{n}$ is nondecreasing on the complementary intervals of $P$, then there is an interval $[l, m]$ containing points of $P$ such that $f_{n}$ is nondecreasing in $[l, m]$.

Proof. Since $f_{n}$ is $[\underline{A C G]}$ on $[a, b],[a, b]$ is a countable union of closed sets on each of which $f_{n}$ is $\underline{A C}$. Let $\left\{E_{i}\right\}$ be the sequence of closed sets such that $[a, b]=\bigcup_{i} E_{i}$ and $f_{n}$ is $\underline{A C}$ on $E_{i}$ for each $i$. Then $P=\bigcup_{i}\left(P \cap E_{i}\right)$. So, by Baire's theorem there is a $i_{0}$ and an interval $[l, m]$ such that $P \cap[l, m] \subset P \cap E_{i_{0}}$. Hence $f_{n}$ is $\underline{A C}$ on $P \cap[l, m]$. Since $f_{n}$ is nondecreasing in the complementary intervals of $P$, and since $f_{n}$ is a Darboux function, $f_{n}$ is continuous and non- 
decreasing in the closure of each contiguous interval of $P$ in $[l, m]$. Hence $f_{n}$ is $\underline{A C}$ on $[l, m]$. Since $\bar{f}_{n+1}^{+} \geqq 0$ almost everywhere in $[l, m]$, the proof is completed by Lemma 9 .

LEmma 11. Let $f$ be $\left[A C_{n} G^{*}\right]$ on $[a, b]$ and let $\bar{f}_{n+1}^{+} \geqq 0$ almost everywhere in $[a, b]$. If $P$ is any perfect subset of $[a, b]$ such that $f_{n}$ is nondecreasing on the complementary intervals of $P$ then there is an interval $[l, m]$ containing points of $P$ such that $f_{n}$ is nondecreasing in $[l, m]$.

Proof. As in the proof of Lemma 10, there is an interval $[l, m]$ such that $f$ is $\underline{A C}_{n}^{*}$ on $P \cap[l, m]$. By Lemma 7 we may suppose that $f_{n}$ is bounded on $P \cap[l, m]$. Also since $f_{n}$ is nondecreasing on the contiguous intervals of $P$ in $[l, m]$, and since $f_{n}$ is a Darboux function, $f_{n}$ is bounded on $[l, m]$. So, by Lemma $1 f_{n}$ is $\underline{A C}$ on $P \cap[l, m]$. To complete the proof one is to apply the argument of Lemma 10.

\section{Properties of generalized derivatives.}

THEOREM 12. Let $f$ be defined in $[a, b]$ and let $f_{n}$ exist on $E \subset$ $[a, b]$. If $\bar{f}_{n+1}<\infty$ on $E$ then $f$ is $\underline{A C_{n} G^{*}}$ on $E$.

Proof. For a positive integer $r$ let $E_{r}$ denote the set of all points $x$ of $E$ such that

$$
0<|t-x| \leqq \frac{1}{r} \text { implies } \frac{\varepsilon_{n}(x, t)}{(t-x) /(n+1)}<r .
$$

Let $E_{r i}=E_{r} \cap[i / r,(i+1) / r]$. Then

$$
E=\bigcup_{r=1}^{\infty} \bigcup_{i=-\infty}^{\infty} E_{r i}
$$

We shall show that $f$ is $\overline{A C}_{n}^{*}$ on each $E_{r i}$. Let $x_{1} \in E_{r i}, x_{2} \in E_{r i}, x_{1}<$ $x_{2}$, and let $x_{1} \leqq t \leqq x_{2}$. Then from (3.1)

$$
\varepsilon_{n}\left(x_{1}, t\right)<\frac{r}{n+1}\left(t-x_{1}\right) \leqq \frac{r}{n+1}\left(x_{2}-x_{1}\right)
$$

and

$$
-\varepsilon_{n}\left(x_{2}, t\right)<\frac{r}{n+1}\left(x_{2}-t\right) \leqq \frac{r}{n+1}\left(x_{2}-x_{1}\right) .
$$

Hence

$$
\sup _{x_{1} \leqq t \leqq x_{2}} \varepsilon_{n}\left(x_{1}, t\right) \leqq \frac{r}{n+1}\left(x_{2}-x_{1}\right), \sup _{x_{1} \leqq t \leqq x_{2}}\left\{-\varepsilon_{n}\left(x_{2}, t\right)\right\} \leqq \frac{r}{n+1}\left(x_{2}-x_{1}\right)
$$


which gives

$$
\bar{\omega}_{n}\left(x_{1}, x_{2}\right) \leqq \frac{r}{n+1}\left(x_{2}-x_{1}\right)
$$

It is now easy to prove that $f$ is $\overline{A C_{n}^{*}}$ on each $E_{r i}$. The proof is completed by (3.2).

By using the analogue of Theorem 12 for $A C_{n} G^{*}$ and the Corollary 4 we get with the help of the Denjoy-Hinčin theorem [7, p. 223].

COROLlaRY 13. If $f_{n}$ exists on $E \subset[a, b]$ and if $-\infty<\underline{f}_{n+1} \leqq$ $\bar{f}_{n+1}<\infty$ on $E$ then $f$ is $A C_{n} G^{*}$ on $E$ and $\left(f_{n}\right)_{a p}^{\prime}$ exists almost everywhere in $E$.

THEOREM 14. If $f_{n}$ exists and is [ACG] in $[a, b]$ and if $\bar{f}_{n+1} \geqq 0$ almost everywhere in $[a, b]$ then $f_{n}$ is continuous and nondecreasing in $[a, b]$.

Proof. Let $G$ be the set of all points $x$ in $[a, b]$ such that there exists a neighborhood of $x$ in which $f_{n}$ is nondecreasing. Then the set $H=[a, b]-G$ is perfect. If possible suppose that $H$ is nonempty. Let $\left\{\left(a_{k}, b_{k}\right)\right\}$ by the set of complementary intervals of $H$ in $[a, b]$. Then $f_{n}$ is nondecreasing in each interval $\left(a_{k}, b_{k}\right)$. By Lemma 10 there is an interval $[l, m]$ containing points of $H$ such that $f_{n}$ is nondecreasing in $[l, m]$. This contradicts the fact that $[l, m]$ contains points of $H$. Thus $H$ is empty and hence $f_{n}$ is nondecreasing in $[a, b]$. The continuity follows from the Darboux property of $f_{n}$.

COROLlary 15. If $f_{n}$ is [ACG] on $[a, b]$ and if one of the derivatives $\bar{f}_{n+1}^{+}, \bar{f}_{n+1}^{-}, f_{n+1}^{+}, f_{n+1}^{-}$vanishes almost everywhere in $[a, b]$, then $f$ is a polynomial of degree at most $n$.

TheOREM 16. If $f$ is $\left[A C_{n} G^{*}\right]$ on $[a, b]$ and if $\bar{f}_{n+1}^{+} \geqq 0$ almost everywhere in $[a, b]$ then $f_{n}$ is continuous and nondecreasing in $[a, b]$.

The proof is similar to that of Theorem 14, but applying Lemma 11 instead of Lemma 10.

Corollary 17. If $f$ is $\left[A C_{n} G^{*}\right]$ on $[a, b]$ and if one of the derivatives $\bar{f}_{n+1}^{+}, \bar{f}_{n+1}^{-}, f_{n+1}^{+}, \underline{f}_{n+1}^{-}$vanishes almost everywhere in $[a, b]$ then $f$ is a polynomial of degree at most $n$.

THEOREM 18. If $f$ is $\left[A C_{n} G^{*}\right]$ on $E \subset[a, b]$ then for almost all $x$ in $E, f_{n+1}(x)$ and $\left(f_{n}\right)_{a p}^{\prime}(x)$ exist and equal each other. 
Proof. We shall follow the argument of Sargent [8] with some essential modifications. Since $f$ is $\left[A C_{n} G^{*}\right]$ on $E$, there is a sequence of closed sets $\left\{Q_{i}\right\}$ such that $E=\bigcup_{i} Q_{i}$ and $f$ is $A C_{n}^{*}$ on each $Q_{i}$. The theorem will therefore be proved if we prove that if $f$ is $A C_{n}^{*}$ on a closed set $Q \subset[a, b]$ then $f_{n+1}$ exists and equals $\left(f_{n}\right)_{a p}^{\prime}$ almost everywhere in $Q$.

Set $g(x)=f(x)-\lambda(x)$, where $\lambda$ is the function obtained by applying Lemma 5 on $Q$. Then

$$
g_{r}(x)=0 \text { for } x \in Q, \quad r=0,1,2, \cdots n .
$$

By Lemma $6, g$ is $A C_{n}^{*}$ on $Q$. Let $\left\{\left(\alpha_{i}, \beta_{i}\right)\right\}$ be the set of complementary intervals of $Q$ in $[a, b]$. Define

$$
\begin{aligned}
h(x) & =0, x \in Q \\
& =\omega_{n}\left(g, \alpha_{i}, \beta_{i}\right), \alpha_{i}<x<\beta_{i} .
\end{aligned}
$$

Since $g$ is $A C_{n}^{*}$ on $Q, h$ is $A C^{*}$ on $Q$. Also $\lambda^{(n+1)}$ is $A C^{*}$ on $Q$ by Lemma 5. Hence $h^{(1)}$ and $\lambda^{(n+1)}$ exist almost everywhere in $Q$. Since $f_{n}=$ $\lambda^{(n)}$ on $Q$, it follows that

$$
\lambda^{(n+1)}(x)=\left(f_{n}\right)_{a p}^{\prime}(x)
$$

holds whenever $x$ is a point of density of $Q$ and $\lambda^{(n+1)}(x)$ exists. By a routine calculation it can be shown that $f_{n+1}(\xi)=\lambda^{(n+1)}(\xi)$ holds whenever $\xi$ is a point of density of the set where $\lambda^{(n)}$ and $h^{(1)}$ exist finitely. This completes the proof.

THEOREM 19. Let $f$ be defined on $[a, b]$ and let $f$ be $A C_{n} G^{*}$ on a measurable set $E \subset[a, b]$. Then for almost all points $x$ in $E, f_{n+1}(x)$ and $\left(f_{n}\right)_{a p}^{\prime}(x)$ exist finitely and equal each other.

Proof. There is a sequence of closed sets $\left\{F_{i}\right\}$ such that

$$
\mu\left(\bigcup_{i} F_{i}\right)=\mu(E) \text {. }
$$

Clearly for a fixed $i, f$ is $A C_{n} G^{*}$ on $F_{i}$ and hence $F_{i}$ can be expressed as the union of a sequence of sets $\left\{F_{i j}\right\}$ such that $f$ is $A C_{n}^{*}$ on $F_{i j}$ for each $j$. By Lemma $2 f$ is $A C_{n}^{*}$ on the closure $\bar{F}_{i j}$ for each $j$. Since

$$
\bar{F}_{i}=F_{i}=\bigcup_{j} \bar{F}_{i j},
$$

$f$ is $\left[A C_{n} G^{*}\right]$ on $F_{i}$. By Theorem $18, f_{n+1}$ and $\left(f_{n}\right)_{a p}^{\prime}$ exist and equal each other at almost all points of $F_{i}$. Hence the result follows from (3.3). 
CoROLlary 20. Let $f$ be measurable on $[a, b]$ and let $f_{n}$ exist finitely on $E \subset[a, b]$ and let

$$
-\infty<\underline{f}_{n+1} \leqq \bar{f}_{n+1}<\infty
$$

hold on $E$. Then for almost all points in $E, f_{n+1}$ and $\left(f_{n}\right)_{a p}^{\prime}$ exist finitely and equal each other.

Proof. Let $G$ be the set of all points in $[a, b]$ where $f_{n}$ exists finitely and the relation (3.4) holds. Then it is sufficient to prove that for almost all points of $G, f_{n+1}$ and $\left(f_{n}\right)_{a p}^{\prime}$ exist finitely and equal each other.

Since

$$
f_{n}(x)=\lim _{u \rightarrow 0} \frac{1}{u^{n}} \sum_{i=0}^{n}(-1)^{n-i}\left(\begin{array}{c}
n \\
i
\end{array}\right) f(x+i u)
$$

$f_{n}$ is measurable. In a similar manner it can be shown that $\bar{f}_{n+1}$ and $f_{n+1}$ are measurable. So, the set $G$ is measurable. Also by Corollary 13, $f$ is $A C_{n} G^{*}$ on $G$. So, applying Theorem $19, f_{n+1}$ and $\left(f_{n}\right)_{u p}^{\prime}$ exists finitely and equal each other at almost all points in $G$.

CoROllary 21. Let $f$ be measurable on $[a, b]$ and let $f_{n}$ exist finitely on a set $E \subset[a, b]$. If for $x \in E$, we have

$$
\begin{aligned}
f(x+t)=f(x)+t f_{1}(x) & +\cdots+\frac{t^{n}}{n !} f_{n}(x) \\
& +\frac{t^{n+1}}{(n+1) !} \gamma(x, t),
\end{aligned}
$$

where $\gamma(x, t)=0(1)$ as $t \rightarrow 0$, then for almost all $x$ in $E, f_{n+1}$ exists and equals $\left(f_{n}\right)_{a p}^{\prime}$.

Corollary 20 sharpens a result of Zygmund [9, Vol. II, p. 77] that if the $n$th derivative $f_{n}$ exists on a set $E$ of positive measure, then for almost all $x$ in $E$

$$
\left(f_{k-1}\right)_{a p}^{\prime}(x)=f_{k}(x), \quad k=2,3, \cdots n .
$$

Corollary 21 offers a simple proof of Lemma 7 of Marcinkiewicz and Zygmund [4] with the aid of which they proved their very interesting main theorem. The original proof of their lemma is long and involves certain results in the theory of Fourier series and analytic functions. Here the proof depends only on the properties of generalized derivatives. The last result also sharpens another result of Zygmund [9, Vol. II, p. 76].

4. The $P^{n}$-integral. The preceding results allow the $P^{n}$-integral, 
$[2,3]$ to be defined in a method introduced by Ridder for the classical Perron integral, the case $n=1$ of the $P^{n}$-integral; [6].

Let $f$ be a finite real valued function on $[a, b]$. Then a continuous function $M$ is called a $\mathscr{P}^{n}$-major function of $f$ if and only if

(a) $M_{r}$ exists and is finite, $1 \leqq r \leqq n-1$;

(b) $\underline{M}_{n}(x) \geqq f(x)$, for almost all $x$;

(c) $M$ is $\left[A C_{n-1} G^{*}\right]$ below on $[a, b]$;

(d) $M_{r}(a)=0,1 \leqq r \leqq n-1$.

In a similar way $\mathscr{P}^{n}$-minor functions can be defined and then by a standard procedure $[2,3,6]$, this leads to a definition of an integral of Perron type-the $\mathscr{P}^{n}$-integral, say.

When $n=1$ this reduces to Ridder's definition of the Perron integral, [6], except that in (c) above Ridder uses $A C G^{*}$ below rather than $\left[A C G^{*}\right]$ below; however for continuous functions, the case of interest here, these two classes coincide so the two definitions are equivalent. Whether it is possible in general, $n \geqq 2$, to use $A C_{n-1} G^{*}$ below rather than $\left[A C_{n-1} G^{*}\right]$ below is an interesting open question; in particular is this weakening of the requirements of Theorem 16 possible?

The properties given in [2] for the $P^{n}$-integral can be obtained in a similar manner for this new integral. However this is unnecessary since as we will see the two integrals are equivalent.

It follows from the above definition that the function $f$ need only be finite, or indeed defined, almost everywhere.

LEMMA 22. If $M$ is any $\mathscr{P}^{n}$-major function of $f, m$ any $\mathscr{P}^{n}$ minor function of $f$ then $M-m$ is n-convex.

Proof. This follows immediately from Theorem 16 above and Corollary 8, [1].

Corollary 23. If $f$ is $\mathscr{P}^{n}$-integrable, $M, m$ as in Lemma 22 then $M-F$ and $F-m$ are $n$-convex.

CoRollary 24. If $f$ is $\mathscr{P}^{n}$-integrable, $F$ its indefinite integral then $F_{r}(x)$ exists, $a \leqq x \leqq b, 1 \leqq r \leqq n-1$.

Both these corollaries follow using the arguments used in [2]; in particular Theorem 10 (c), (d) and Corollary 11 of [2].

The following simple lemma that can be proved similarly for any integral of Perron type does not seem to have been proved before.

LEMma 25. If $f$ is $\mathscr{P}^{n}$-integrable with zero as its indefinite integral then $f(x)=0$ for almost all $x$. 
Proof. The case $n=1$ is slightly different but the general proof can easily be adapted to this case; we assume then that $n \geqq 2$.

From Corollary 23 every $\mathscr{P}^{n}$-major function $M$ of $f$ is $n$-convex and so $M^{(n)}$ exists almost everywhere, [1, Corollary 15].

Suppose that $f>0$ on some set of positive measure then for some $\alpha, \beta, a<\alpha<\beta<b$ and some $k>0, \varepsilon>0$ there is a set $A \subset[\alpha, \beta]$ such that $\mu(A)>\varepsilon$ and $x \in A$ implies $f(x)>k$.

If $M$ is any $\mathscr{P}^{n}$-major function of $f$ then by Corollary $23, M$ is $n$-convex and so $M^{(n)}$ exists almost everywhere, [1, Corollary 15]. Let $B=\left\{x ; M^{(n)}(x) \geqq k\right\}$; then by (b) in the above definition of $\mathscr{P}^{n}$-major function $A \subset B$ and so

$$
\mu(B)>\varepsilon \text {. }
$$

However, since $M$ is $n$-convex, by Theorem 12 of [1], we have that

$$
\mu(B) \leqq \frac{2 n}{k}\left\{M^{(n-1)}(\beta)-M^{(n-1)}(\alpha)\right\} .
$$

But $M$ can be chosen so that $M(\beta)$ can be made arbitrarily small and so by Corollary 8 (b), [1], the right-hand side of (4.2) can, by the right choice of $M$ be made less than $\varepsilon$.

This contradiction of inequality (4.1) prove that $f(x) \leqq 0$ almost everywhere.

In a similar way it can be shown that $f(x) \geqq 0$ almost everywhere, which completes the proof.

COROLlaRY 26. If $f$, and $g$ are both $\mathscr{P}^{n}$-integrable with the same indefinite integrals then $f(x)=g(x)$ for almost all $x$.

THEOREM 27. The $\mathscr{P}^{n}$-integral is equivalent to the $P^{n}$-integral defined in [2].

Proof. (a) Suppose $f$ is $P^{n}$-integrable. Then by Theorem 22, [2], $f$ is $D^{n}$-integrable. That is to say, there is a function $F$ such that $F$ is $A C_{n-1} G^{*}$ on $[a, b], F_{r}(a)=0,1 \leqq r \leqq n-1$, and $F_{n}(x)=f(x)$ almost everywhere.

By Lemma 5, [8], (also repeated in Lemma 17 (c), [2]), $F$ is $\left[A C_{n-1} G^{*}\right]$ on $[a, b]$ and so is both a $\mathscr{P}^{n}$-major and a $\mathscr{P}^{n}$-minor function of $f$. Hence $f$ is $\mathscr{P}^{n}$-integrable with $F$ as its indefinite $\mathscr{P}^{n}$-integral; but by Theorem 22, [2], $F$ is also the indefinite $P^{n}$-integral of $f$.

(b) Now suppose $f$ is $\mathscr{P}^{n}$-integrable with indefinite integral $F$. Proceeding as in part (a) of the proof of Theorem 22, [2], it follows that $F$ is $\left[A C_{n-1} G^{*}\right]$ on $[a, b]$. (Due to hypothesis (c) of the $\mathscr{P}^{n}$-major functions the proof here is easier as there is no need to appeal to 
either Lemma 21, [2], - Theorem 12 above, - or to Lemma 5, [8].)

By Theorem 18 above $F_{n}$ exists almost everywhere and so from the above definition, $F_{n}$ is $D^{n}$-integrable with $F$ as its indefinite $D^{n}$ integral. By part (a) above then $F_{n}$ is $\mathscr{P}^{n}$-integrable with $F$ as its indefinite $\mathscr{P}^{n}$-integral. Hence by Corollary 26, $F_{n}(x)=f(x)$ almost everywhere; that is to say, $f$ is $D^{n}$-integrable, and so $P^{n}$-integrable with $F$ its indefinite $P^{n}$-integral; this completes the proof.

The critical step in being able to construct an integral of Perron type is a result analogous to Lemma 22. As a result it is possible to vary the definition given above of major functions to obtain different $n$th order Perron integrals.

Firstly we could replace hypothesis (c) by

$M_{n-1}$ is $[A C G]$ below on $[a, b]$.

By appealing to Theorem 14 (rather than Theorem 16 as in proof of Lemma 22) the analogue of Lemma 22 still holds; so in the usual way we define a new integral the $H^{n}$-integral, say. The case $n=1$ of this new integral is equivalent to Ridder's definition of a Perron integral equivalent to the Hinčin totalization, [6, Section 6]; the difference as before is in the use of $[A C G]$ below rather than $A C G$ below. The relation of this new integral to the $P^{n}$-integral is not known since at present no relationship is known between $M_{n-1}$ being [ACG] below and $M$ being $\left[A C_{n-1} G^{*}\right]$ below, other than that given by Corollary 4 .

A second possibility is to replace (c) by (c)* but in addition to replace (b) by

$$
\underline{M}_{n, a p}(x) \geqq f(x) \text { for almost all } x \text {. }
$$

Again appealing to Theorem 14, (noting that $\bar{g}_{n}^{+}(x) \geqq \underline{g}_{n, a p}(x)$ for all $x$ ) the analogue of Lemma 22 still holds; so in the usual way a new integral can be defined. The relation of this integral to the other integrals introduced here, and to the general Denjoy integral, when $n=1$, will be taken up in a later paper.

\section{REFERENCES}

1. P. S. Bullen, $A$ criterion for n-convexity, Pacific J. Math., 36 (1971), 81-98.

2. - The $P^{n-i n t e g r a l, ~ B u l l . ~ A u s t r a l i a n ~ M a t h . ~ S o c ., ~} 14$ (2) (1972), 219-236.

3. R. D. James, Generalized $n$th primitives, Trans. Amer. Math. Soc., 76 (1954), 149-176.

4. J. Marcinkiewicz and A. Zygmund, On the differentiability of functions and summability of trigonometric series, Fund. Math., 26 (1936), 1-43.

5. H. W. Oliver, The exact Peano derivative, Trans. Amer. Math. Soc., 76 (1954), 444-456.

6. J. Ridder, Uber der Perronschen Integralbegriff und seine Beziehung zu der $\mathscr{R}$-, $\mathscr{L}$-, und $\mathscr{D}$-Integralen, Math, Zeit., 34 (1931), 234-239.

7. S. Saks, Theory of the Integral, Warsaw, 1937.

8. W. L. C. Sargent, On generalized derivatives and Cesaro-Denjoy integrals, Proc. 
London Math. Soc., (2) 52 (1951), 365-376.

9. A. Zygmund, Trigonometric Series, Cambridge, 1968.

Received May 1, 1972. The work of this author was supported by the National Research Council of Canada, Grant no. 67-2986.

University of British Columbia 


\section{PACIFIC JOURNAL OF MATHEMATICS}

\section{EDITORS}

D. Gilbarg and J. Milgram

Stanford University

Stanford, California 94305

R. A. Beaumont

University of Washington

Seattle, Washington 98105
J. DUGUNDJI* Department of Mathematics

University of Southern California Los Angeles, California 90007

RICHARD ARENS

University of California

Los Angeles, California 90024

\section{ASSOCIATE EDITORS}

E. F. BeCKenbach

B. H. NeUMaNN

F. WOLF

K. Yoshida

\section{SUPPORTING INSTITUTIONS}

UNIVERSITY OF BRITISH COLUMBIA

UNIVERSITY OF SOUTHERN CALIFORNIA

CALIFORNIA INSTITUTE OF TECHNOLOGY

UNIVERSITY OF CALIFORNIA

MONTANA STATE UNIVERSITY

STANFORD UNIVERSITY

UNIVERSITY OF TOKYO

UNIVERSITY OF NEVADA

UNIVERSITY OF UTAH

NEW MEXICO STATE UNIVERSITY

WASHINGTON STATE UNIVERSITY

OREGON STATE UNIVERSITY

UNIVERSITY OF OREGON

OSAKA UNIVERSITY

UNIVERSITY OF WASHINGTON



* C. DePrima will replace J. Dugundji until August 1974. 


\section{Pacific Journal of Mathematics}

\section{Vol. 47, No. $1 \quad$ January, 1973}

K. Adachi, Masuo Suzuki and M. Yoshida, Continuation of holomorphic

mappings, with values in a complex Lie group ....................

Michael Aschbacher, A characterization of the unitary and symplectic groups



Larry Eugene Bobisud and James Calvert, Energy bounds and virial theorems for abstract wave equations....................................

Christer Borell, A note on an inequality for rearrangements ................

Peter Southcott Bullen and S. N. Mukhopadhyay, Peano derivatives and general

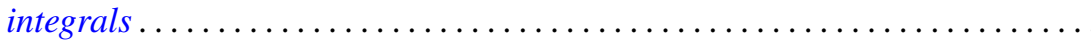

Wendell Dan Curtis, Yu-Lee Lee and Forrest Miller, A class of infinite dimensional subgroups of $\operatorname{Diff}^{r}(X)$ which are Banach Lie groups .........

Paul C. Eklof, The structure of ultraproducts of abelian groups ...............

William Alan Feldman, Axioms of countability and the algebra $C(X) \ldots \ldots \ldots$

Jack Tilden Goodykoontz, Jr., Aposyndetic properties of hyperspaces...........

George Grätzer and J. Płonka, On the number of polynomials of an idempotent algebra. II ...........................................

Alan Trinler Huckleberry, The weak envelope of holomorphy for algebras of

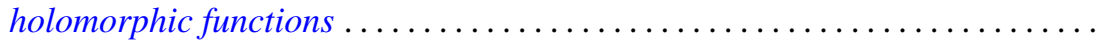

John Joseph Hutchinson and Julius Martin Zelmanowitz, Subdirect sum decompositions of endomorphism rings . . . . . . . . . . . . . . . .

Gary Douglas Jones, An asymptotic property of solutions of

$y^{\prime \prime \prime}+p y^{\prime}+q y=0$.

Howard E. Lacey, On the classification of Lindenstrauss spaces .

Charles Dwight Lahr, Approximate identities for convolution measure algebras.

George William Luna, Subdifferentials of convex functions on Banach

spaces.

Nelson Groh Markley, Locally circular minimal sets. .

Robert Wilmer Miller, Endomorphism rings of finitely generated projective modules

Donald Steven Passman, On the semisimplicity of group rings of linear



Bennie Jake Pearson, Dendritic compactifications of certain dendritic spaces.

Ryōtarō Satō, Abel-ergodic theorems for subsequences ...... .

Henry S. Sharp, Jr., Locally complete graphs. . .

Harris Samuel Shultz, A very weak topology for the Mikusinski field of

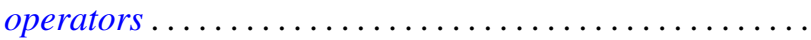

Elena Stroescu, Isometric dilations of contractions on Banach spaces ...

Charles W. Trigg, Versum sequences in the binary system ... . .

William L. Voxman, On the countable union of cellular decompositions of n-manifolds 